\title{
Fractal dimensions of niobium oxide films probed by protons and lithium ions
}

\author{
Esat Pehlivan \\ Department of Physics, Faculty of Arts and Sciences, Istanbul Technical University, Maslak, \\ 34469 Istanbul, Turkey and The Angström Laboratory, Department of Engineering Sciences, \\ Uppsala University, P.O. Box 534, SE-751 21 Uppsala, Sweden \\ Gunnar A. Niklasson ${ }^{\text {a) }}$ \\ The Angström Laboratory, Department of Engineering Sciences, Uppsala University, P.O. Box 534, \\ SE-751 21 Uppsala, Sweden
}

(Received 13 January 2006; accepted 19 June 2006; published online 7 September 2006)

Cyclic voltammetry (CV) and atomic force microscopy (AFM) were used to determine fractal surface dimensions of sputter deposited niobium pentoxide films. Peak currents were determined by $\mathrm{CV}$ measurements. Power spectral densities obtained from AFM measurements of the films were used for calculating length scale dependent root mean square roughness. In order to compare the effect of $\mathrm{Li}$ and $\mathrm{H}$ ion intercalation at the fractal surfaces, $\mathrm{LiClO}_{4}$ based as well as propionic acid electrolytes were used. The $\mathrm{CV}$ measurements gave a fractal dimension of 2.36 when the films were intercalated by $\mathrm{Li}$ ions and 1.70 when the films were intercalated by protons. AFM measurements showed that the former value corresponds to the fractal surface roughness of the films, while the latter value is close to the dimensionality of the distribution of hillocks on the surface. We conclude that the protons are preferentially intercalated at such sites. (c) 2006 American Institute of Physics. [DOI: $10.1063 / 1.2337164]$

\section{INTRODUCTION}

Niobium oxide films are widely used for optical coatings because of their high refractive index and dielectric constant. ${ }^{1}$ Other applications include batteries, solar energy devices, ${ }^{2}$ oxygen sensors, and display devices. ${ }^{3}$ After the electrochromic behavior of niobium pentoxide was reported ${ }^{4}$ it became an extensively studied cathodic electrochromic material. Contrary to most other electrochromic materials, well-crystallized niobium pentoxide films show a wider optical modulation range and a better durability than amorphous films. ${ }^{5}$ Niobium pentoxide films, when intercalated by ions from an electrolyte, exhibit different colors depending on their crystallinity. It has been found that crystalline films exhibit a blue and amorphous ones exhibit a brown color. ${ }^{6}$ These features make niobium pentoxide different from other electrochromic materials. The dynamics of the intercalation process and the electrochromic switching depends on the possibly fractal structure of the film surface. ${ }^{7}$ The fractal structure of electrochromic niobium pentoxide films has never been studied before. In this work, fractal surface properties of sputter coated niobium pentoxide films have been investigated by cyclic voltammetry $(\mathrm{CV})$ and atomic force microscopy (AFM) measurements. Remarkably, the CV results show major differences depending on whether protons or $\mathrm{Li}$ ions are used to probe the surface.

\section{EXPERIMENT}

The films were deposited using a Balzers UTT $400 \mathrm{dc}$ magnetron sputtering system equipped with a turbomolecular

\footnotetext{
${ }^{a)}$ Author to whom correspondence should be addressed; electronic mail: gunnar.niklasson@angstrom.uu.se
}

pump. Niobium metal targets with a diameter of $5 \mathrm{~cm}$ and a purity of more than $99.95 \%$ were used. Sputtering took place in a mixture of argon and oxygen gases with purity of $99.998 \%$. The background pressure was about $10^{-6}$ Torr after at least $8 \mathrm{~h}$ of baking at $130{ }^{\circ} \mathrm{C}$ before deposition. Deposition took place at $35 \mathrm{mTorr}$ total pressure. After backfilling the chamber with argon gas, the substrate and niobium target were presputtered to clean the surface, and then argon and oxygen gases were introduced into the chamber. The flow rates of argon and oxygen gases were 4.5 and 100 SCCM (SCCM denotes cubic centimeter per minute at STP), respectively. Indium tin oxide coated glass slides with a sheet resistance of $60 \Omega /$ sq were used as substrates and positioned $13 \mathrm{~cm}$ from the target. The topography of the films was analyzed by a Nanoscope III AFM equipped with a vibration absorbing board and operated in tapping mode. Images with $512 \times 512$ pixels were acquired using various scan lengths from $0.5-5 \mu \mathrm{m}$. Coloration and bleaching processes were controlled by an Autolab PGSTAT 10 potentiostat. A three electrode cell was used and the surface under study was employed as the working electrode. Two different configurations were used for $\mathrm{CV}$ measurements. In the first configuration, $1 M \mathrm{LiClO}_{4}$ dissolved in propylene carbonate (PC) was used as the electrolyte. Lithium foils were used as both the reference and the counterelectrode. In the second case, $1 M$ propionic acid was used as electrolyte. Platinum and $\mathrm{Ag} / \mathrm{AgCl}$ electrodes were used as counterelectrode and reference electrode, respectively. All CV measurements were carried out in a glovebox containing argon gas with low humidity. During all measurements the dew point of the glovebox was $-52{ }^{\circ} \mathrm{C}$ which corresponds to $30 \mathrm{ppm}$ of water. The films were subjected to at least five precycles until stable 
voltammetric cycles were achieved. In order to get a wide range in the plot of peak current versus scan rate, various scan rates from 10 to $500 \mathrm{mV} / \mathrm{s}$ were used.

\section{ANALYSIS OF THE FRACTAL SURFACE}

The electrochemical reactions that we consider take place at the interface between the film and the electrolyte. In addition, many properties of thin films depend on the topography of the surface. Hence it is very important whether the surface is rough or smooth, porous or nonporous. Fractal dimension analysis gives important information about the structure of the surface. Various physical and chemical ${ }^{8-13}$ methods have been used for determination of fractal dimension. Two of the most important methods for calculating fractal dimension are $\mathrm{CV}$ and $\mathrm{AFM}$.

A well-known equation established in 1948 by Randles ${ }^{14}$ and Sevcik ${ }^{15}$ independently from each other shows that the peak current in a $\mathrm{CV}$ measurement is proportional to the square root of scan rate. Hence the slope of a log-log plot of the peak current versus scan rate is expected to be 0.5 .

A method for determining fractal dimension from $\mathrm{CV}$ measurements under diffusion limited conditions was introduced and an improved Randles-Sevcik equation for fractal surfaces was obtained. ${ }^{16} \mathrm{~A}$ simplified form of the improved Randles-Sevcik equation can be found:

$$
I_{p}=\frac{\lambda_{o}^{2 \alpha-1}(n F)^{\alpha+1} A(\pi D)^{1-\alpha} C \chi_{\max }(\alpha)}{\sin (\pi \alpha)(R T)^{\alpha}} \nu^{\alpha},
$$

where $I_{p}$ is the peak current, $\lambda_{0}$ is the length corresponding to the outer cutoff of the fractal structure, $\alpha$ is the fractal parameter, $n$ is the number of transferred electrons per atom, $F$ is Faraday's constant, $A$ is the area of the electrode, $D$ is the diffusion coefficient, $C$ is the concentration of the electrolyte, $\chi$ is a dimensionless function of $\alpha, R$ is the molar gas constant, $T$ is the temperature, and $\nu$ is the scan rate. It can be seen from Eq. (1) that the peak current $I_{p}$ is directly related to the fractal parameter $\alpha$ and Eq. (1) can be simplified to $I_{p} \sim \nu^{\alpha}$ when the other parameters are kept constant. The fractal parameter can be found from the slope of a log-log plot of the peak current versus scan rate.

It was shown, for diffusion to a fractal surface, that $\alpha$ and the fractal dimension $d_{f}$ of the analyzed surface are connected by

$$
\alpha_{1}=\left(d_{f 1}-1\right) / 2,
$$

where subscript 1 denotes the first method, i.e., CV. After calculating the fractal parameter, Eq. (1) can also be used for calculating the diffusion coefficient $D$, since the only remaining unknown parameter is $\lambda_{0}$, and this can be obtained from AFM measurements (see below).

The second method to obtain the fractal dimension of the surface is from AFM. The scaling of surface roughness as a function of lateral length scale has been studied before. ${ }^{17}$ The roughness of a fractal surface can be described by power laws, including a roughness exponent $\alpha_{2}$. The fractal dimension of the surface is given by

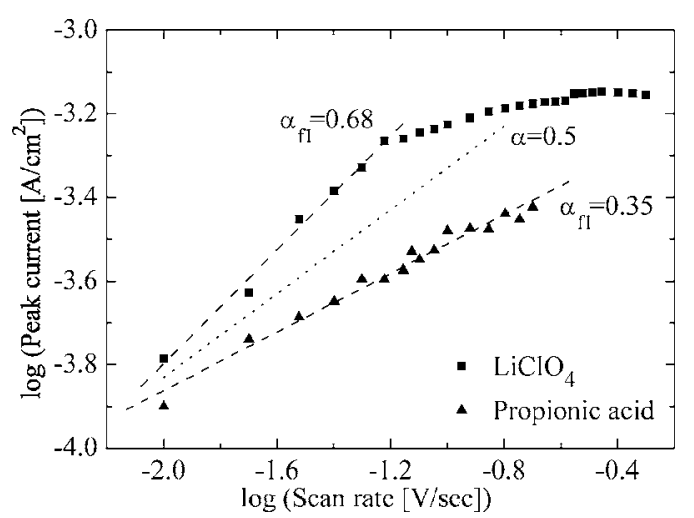

FIG. 1. Log-log plot of peak current vs scan rate for sputter deposited niobium pentoxide film when $\mathrm{LiClO}_{4}$ and propionic acid were used as electrolytes. Dashed lines are linear fits to measured data and the dotted line has a slope of 0.5. The regression coefficient (chi square) of the power-law fits was 0.995 .

$$
d_{f 2}=3-\alpha_{2}
$$

and it is independent of film thickness. Subscript 2 denotes the second method, i.e., AFM. The rms roughness values of the films are given by the square root of the integral of the power between selected points, ${ }^{18}$ that is,

$$
\mathrm{rms}=\left(\int_{L_{\min }}^{L_{\max }} W(p, q) d p d q\right)^{1 / 2},
$$

where $W(p, q)$ is the power of the AFM image at the coordinate $(p, q)$ and the power is the roughness amplitude squared. Maximum values of $p$ and $q$ depend on the resolution of the image. In order to obtain the rms roughness, the two dimensional isotropic PSD function of the film was used. Subsequently, length scale dependent rms values were obtained by changing the upper limit of the integral. The analysis was carried out for various scan lengths. AFM measurements at scan lengths larger than $5 \mu \mathrm{m}$ were not used since they showed no self-similar behavior and gave no information about the fractal dimension. Similarly, AFM measurements with scan lengths below $0.5 \mu \mathrm{m}$ were not used since the resolution becomes close to the dimension of the AFM tip and this causes large errors.

\section{RESULTS AND DISCUSSION}

\section{A. $\mathrm{LiClO}_{4}$ as electrolyte}

Figure 1 shows the log-log plot of peak current versus scan rate for both $\mathrm{LiClO}_{4}$ and propionic acid electrolytes. In Fig. 1, dashed lines show linear fits to the measured data and the dotted line with a slope of 0.5 was drawn for comparison. The fractal parameter of sputter deposited niobium pentoxide films in the $\mathrm{LiClO}_{4}-\mathrm{PC}$ electrolyte was found to be 0.68 . At scan rates higher than $60 \mathrm{mV} / \mathrm{s}$, the curve starts to become flatter. This behavior could be due to the upper cutoff to the fractal region; in addition diffusion limited conditions might not be valid anymore. If the scan rate was increased to very high values, e.g., more than $250 \mathrm{mV} / \mathrm{s}$, the sample could not respond to such fast scans and the same peak current was 


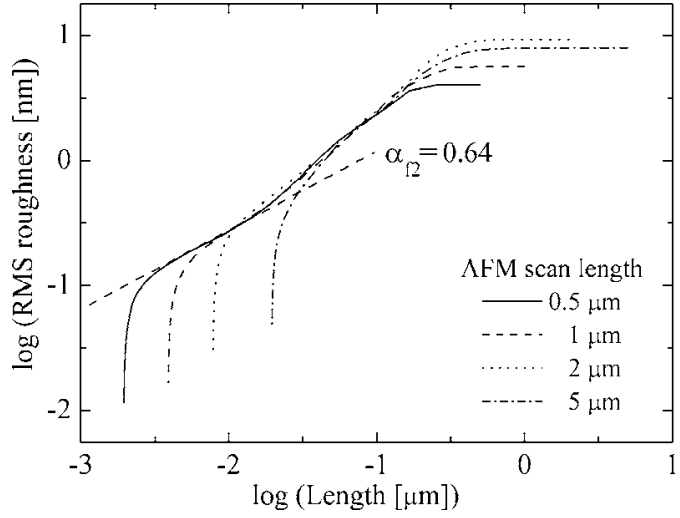

FIG. 2. Log-log plot of rms roughness values for sputter deposited niobium pentoxide films as a function of length scale. Curves extracted from AFM images taken with various scan lengths are shown. The scan lengths are given in the inset.

observed for various rates. We found from Eq. (2) that our calculated fractal parameter of 0.68 corresponds to a fractal dimension of 2.36 .

Figure 2 shows the rms roughness as a function of length scale for our niobium oxide films. Data from images taken with different scan lengths are shown. It is seen from Fig. 2 that the rms roughness is constant for large length scales close to the image size and it drops drastically at small length scales due to the resolution of the images. The rms roughness value of an image taken with higher resolution (smaller scan length) drops at a shorter length scale. In the middle region, all the curves (and their envelope) exhibit two power-law regions with different exponents. The slope of the curves is unity at length scales larger than $16 \mathrm{~nm}$ and decreases for length scales below this value. It was found that the latter region has a slope of 0.64 , which corresponds to a fractal dimension of 2.36. This result is the same as obtained by the $\mathrm{CV}$ measurements.

The outer cutoff of the sample was found from the crossover between the two power-law regions in Fig. 2 to be $16 \mathrm{~nm}$. The inner cutoff is not clearly seen in Fig. 2, but it appears that the fractal region is less than a decade. Using the value for the upper cutoff in Eq. (1), the diffusion coefficient of the $\mathrm{Li}$ ions was found to be $3.25 \times 10^{-10} \mathrm{~cm}^{2} / \mathrm{s}$ which is in very good agreement with previous data for pulsed laser deposited niobium oxide films. ${ }^{19}$

\section{B. Propionic acid as electrolyte}

The fractal parameter of the film, when propionic acid was used as electrolyte, was found from Fig. 1 to be 0.35 which is smaller than 0.5 . This corresponds to a fractal dimension of 1.70 . It has been shown previously that a fractal dimension smaller than 2 can characterize a partially active surface, for example, exhibiting well-defined "hillocks" as reaction sites. ${ }^{20,21}$ In order to test this hypothesis, we carried out a fractal analysis of the hillock distribution on the surface. For this purpose, a $30 \times 30 \mu \mathrm{m}$ AFM image of the sample was taken. The scan area was as large as possible in order to get better statistics. Figure 3 shows the hillocks in an AFM image, where we take all protrusions higher than $25 \%$ of the maximum height to be defined as hillocks. The num-

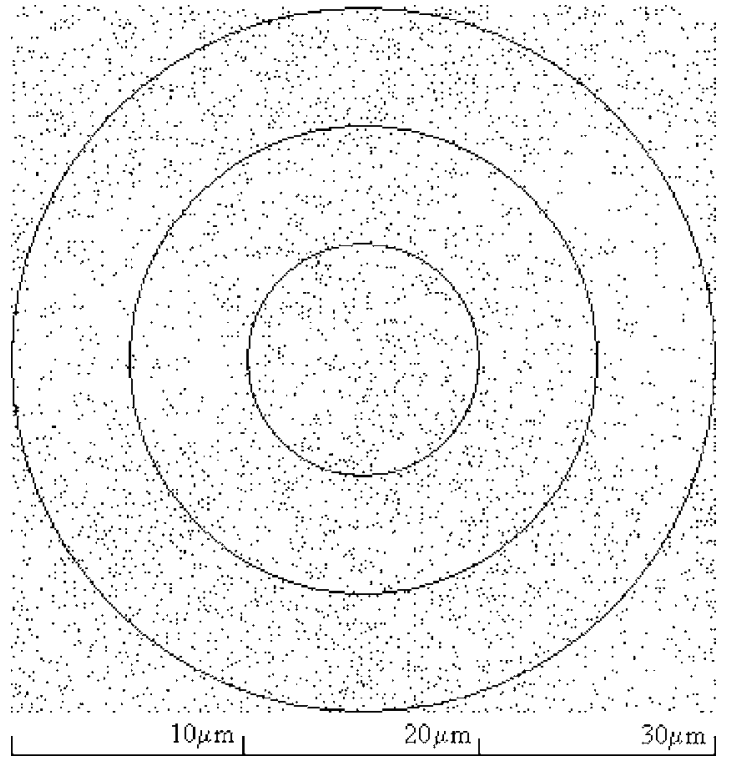

FIG. 3. The distribution of hillocks on a $30 \times 30 \mu \mathrm{m}$ AFM image. All protrusions higher than $25 \%$ of the maximum height are shown.

ber of hillocks inside circles of various radii on the image was determined; for example, circles of 5, 10, and $15 \mu \mathrm{m}$ radius are shown in Fig. 3. Figure 4 shows the result of the hillock analysis. The slope of the linear fit directly gives the fractal dimension and it was found to be 1.77. It is close to the value obtained from the CV measurement when propionic acid was used as an electrolyte.

\section{CONCLUSIONS}

We have determined fractal dimensions of sputter deposited niobium pentoxide films when $\mathrm{Li}$ and $\mathrm{H}$ ions are intercalated. Two different methods, CV and AFM, were used. Remarkably, the results from CV were totally different in the cases of $\mathrm{Li}^{+}$and $\mathrm{H}^{+}$intercalation. When the films were intercalated with $\mathrm{Li}$ ions in a $\mathrm{LiClO}_{4}$ electrolyte, their fractal dimension as obtained from $\mathrm{CV}$ of 2.36 corresponds to the dimension of surface roughness obtained from AFM. However, when the films were intercalated by protons in propionic acid, a dimension of 1.70 was found from CV. Obviously the protons are not penetrating the whole surface but are rather intercalated at special "reaction sites." We identify

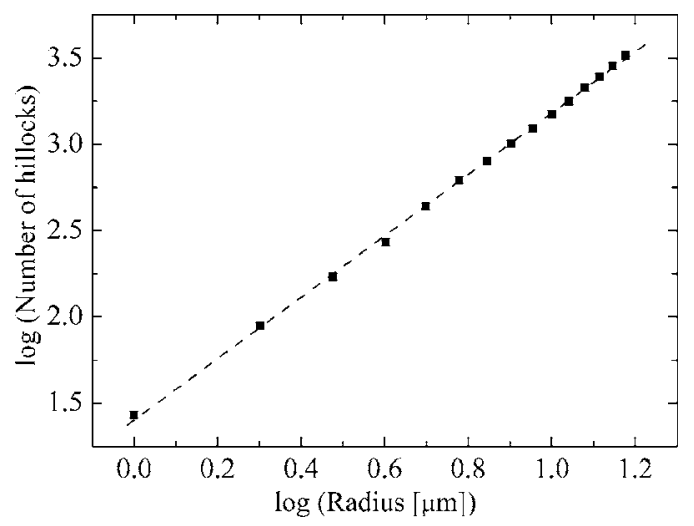

FIG. 4. Log-log plot of number of hillocks vs radius. A linear fit (dashed line) gives the fractal dimension of 1.77 . 
these sites as hillocks fractally distributed over the surface. The dimensionality of the hillock distribution was found from AFM to be close to the CV value.

\section{ACKNOWLEDGMENTS}

This work was partially supported by a grant from the Swedish Research Council. One of the authors (E.P.) gratefully acknowledges Professor C. G. Granqvist, Professor Fatma Tepehan, and Professor Galip Tepehan for making this scientific collaboration possible.

${ }^{1}$ J. J. Van Gladbeek and R. E. Van De Leest, Thin Solid Films 201, 137 (1991).

${ }^{2}$ M. A. Aegerter, Sol. Energy Mater. Sol. Cells 68, 401 (2001).

${ }^{3}$ P. M. S. Monk, R. J. Mortimer, and D. R. Rosseinsky, Electrochromism: Fundamentals and Applications (VCH, Weinheim, 1995).

${ }^{4}$ B. Reichman and A. J. Bard, J. Electrochem. Soc. 127, 241 (1980).

${ }^{5}$ K. Yoshimura, T. Miki, S. Iwama, and S. Tanemura, Thin Solid Films 281-282, 235 (1996).

${ }^{6}$ A. Pawlicka, M. Atik, and M. A. Aegerter, Thin Solid Films 301, 236 (1997).

${ }^{7}$ M. Stromme Mattsson, G. A. Niklasson, and C. G. Granqvist, Phys. Rev.
B 54, 2968 (1996).

${ }^{8}$ J. Chevrier, V. Le Thanh, R. Buys, and J. Derrien, Europhys. Lett. 16, 737 (1991).

${ }^{9}$ J. E. Martin and A. J. Hurd, J. Appl. Crystallogr. 20, 61 (1987).

${ }^{10}$ D. Avnir, D. Farin, and P. Pfeifer, Nature (London) 308, 261 (1984).

${ }^{11}$ R. Chiarello, V. Panella, J. Krim, and C. Thompson, Phys. Rev. Lett. 67, 3408 (1991).

${ }^{12}$ J. M. Gómez-Rodriguez, A. Asenjo, R. C. Salvarezza, and A. M. Baro, Ultramicroscopy 42-44, 1321 (1992).

${ }^{13}$ G. L. M. K. S. Kahanda and M. Tomkiewicz, J. Electrochem. Soc. 137, 3423 (1990)

${ }^{14}$ J. E. B. Randles, Trans. Faraday Soc. 44, 327 (1948).

${ }^{15}$ A. Sevcik, Collect. Czech. Chem. Commun. 13, 349 (1948).

${ }^{16}$ M. Strømme, G. A. Niklasson, and C. G. Granqvist, Solid State Commun. 96, 151 (1995).

${ }^{17}$ A.-L. Barabasi and H. E. Stanley, Fractal Concepts in Surface Growth (Cambridge University Press, England, 1995).

${ }^{18}$ Nanoscope Command Reference Manual, Version 4.42 (Digital Instruments, Sta. Barbara, CA, 1999).

${ }^{19}$ Z.-W. Fu, J.-J. Kong, and Q.-Z. Quin, J. Electrochem. Soc. 146, 3914 (1999).

${ }^{20}$ M. Stromme Mattsson, G. A. Niklasson, and C. G. Granqvist, Phys. Rev. B 54, 17884 (1996).

${ }^{21}$ T. Pajkossy and L. Nyikos, Electrochim. Acta 34, 171 (1989). 